Energy Sources, 25:357-370, 2003

Copyright (C) 2003 Taylor \& Francis

$0090-8312 / 03 \$ 12.00+.00$

DOI: $10.1080 / 00908310390142389$

\title{
Global Warming: Are We Confusing Cause and Effect?
}

\author{
LEONID F. KHILYUK \\ GEORGE V. CHILINGAR \\ Department of Civil Engineering \\ University of Southern California \\ Los Angeles, California
}

\begin{abstract}
The writers show that the present-day global warming is not due to the increase in the volume of greenhouse gases, but rather to the increased solar activity. It appears that we are at the rising phase of the latest 80-90 year cycle of the solar activity. At the present time, there is no sound justification for the cut in the man-induced carbon dioxide emission as required by the Kyoto Protocol of 1997. The rising global temperature drives large volumes of $\mathrm{CO}_{2}$ from the ocean water into the atmosphere. Thus "cause" and "effect" of global warming phenomenon are misunderstood by many scientists.
\end{abstract}

\section{Established Facts About Climatic Changes}

The main characteristic of the Earth's climate is temperature. During the 20th Century, the global mean surface temperature has increased by approximately $1^{\circ} \mathrm{F}$ (Figure 1 ). "The 20th Century's warmest years all occurred in the last 15 years of the century. Of these, 1998 was the warmest year on record. The snow cover in the Northern Hemisphere and floating ice in the Arctic Ocean have decreased. Globally, sea level has risen 4-8 inches over the past century. Worldwide precipitation over land has increased by about $1 \%$. The frequency of extreme rainfall events has increased throughout much of the United States" (EPA). Over the same period of time, the concentrations of water vapor, carbon dioxide, methane, and other greenhouse gases in the atmosphere increased, partly as a result of the human utilization of coal, oil, and natural gas. Increasing concentrations of carbon dioxide, for example, is illustrated in Figure 2.

\section{Simplistic Explanation of Climatic Changes}

Energy radiated from the sun heats the Earth's surface and determines the Earth's climate. In turn, heated Earth reradiates (at a longer wave length) part of this energy back into space. Some of this reradiated energy is absorbed and reemitted in all directions by the molecules of greenhouse gases in the atmosphere. If the concentration of the greenhouse gases in the atmosphere increases, then the portion of the trapped energy increases, heating the Earth and changing the Earth's climate. A schematic diagram of interaction

Received 19 October 2002; accepted 24 November 2002.

Address correspondence to Dr. George V. Chilingar, 101 S. Windsor Blvd., Los Angeles, CA 90004. E-mail: gchiling@usc.edu 


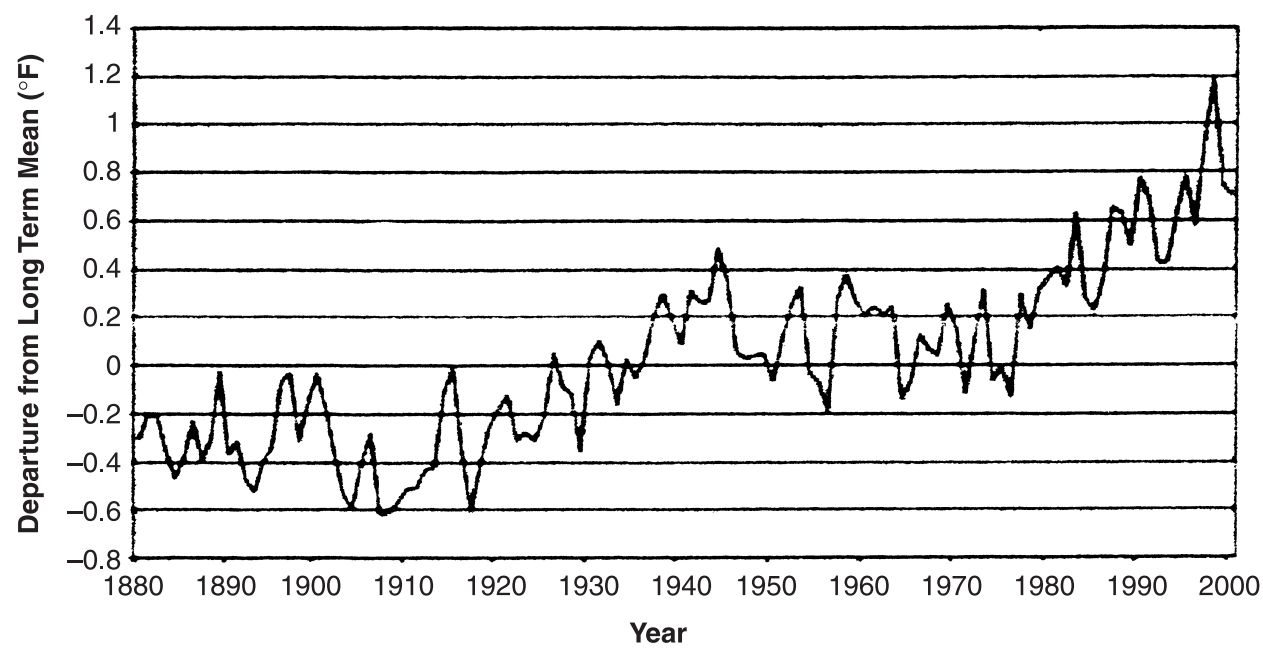

Figure 1. Global average temperature changes in the twentieth Century. (Modified from the EPA Global Warming Site: Climate. U.S. National Climatic Data Center, 2001.)

between the solar radiation and the greenhouse gases is shown in Figure 3. A commonly used term for this phenomenon is the "greenhouse effect." According to many scientists, without the greenhouse effect, present-day Earth's global temperature would be only $5^{\circ} \mathrm{F}$ instead of $59^{\circ} \mathrm{F}$. This phenomenon has been going on throughout the Earth's history over the past four billion years and has been known about for approximately 200 years. The great French mathematician Fourier described it first in 1822 (Mester, 1996). At the end of the nineteenth Century, Arrhenius (1896) presented a hypothesis on heating

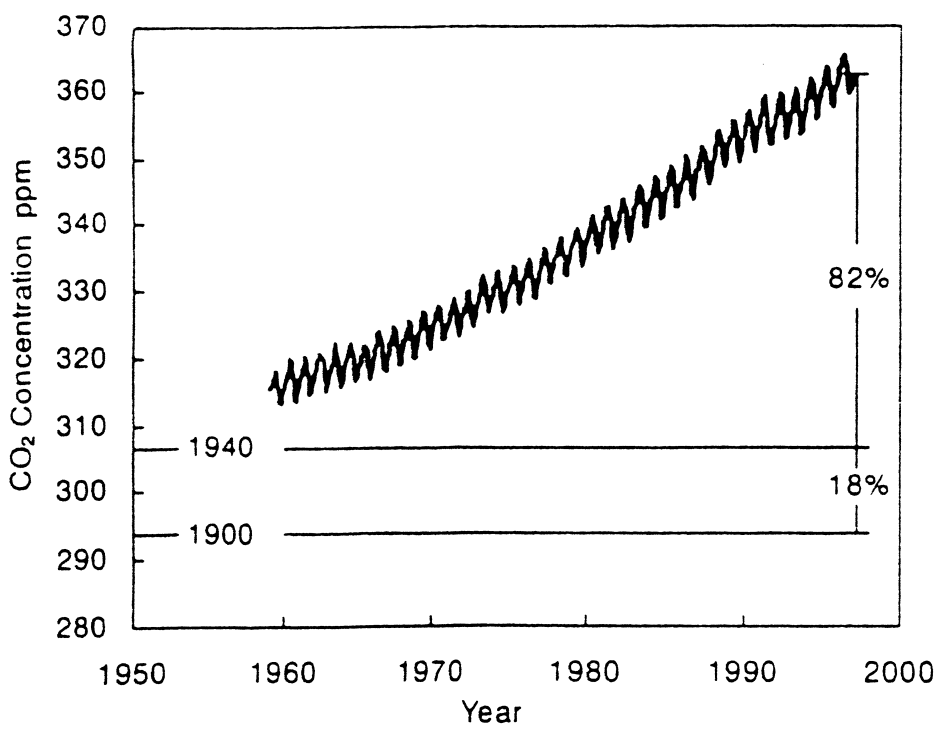

Figure 2. Changes in atmospheric $\mathrm{CO}_{2}$ concentration in parts per million (ppm) at Mauna Loa, Hawaii. (Modified from Keeling and Whorf, 1997.) Approximate global level of atmospheric $\mathrm{CO}_{2}$ in 1900 and 1940 is also shown. 


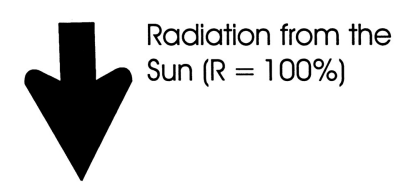

Thermosphere

Mesopause $(\approx 80 \mathrm{~km})$

Mesosphere

Stratopause $(\approx 50-55 \mathrm{~km})$

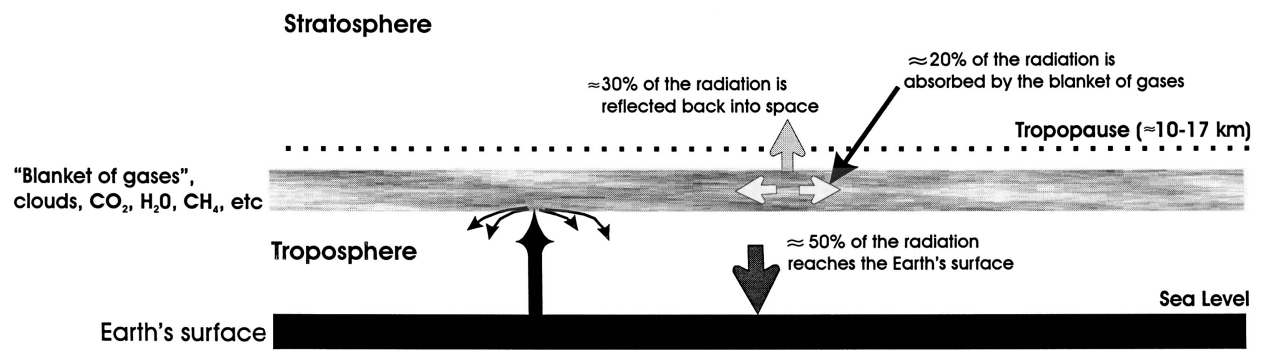

Figure 3. The greenhouse effect. (Modified from the World Resources Institute, Changing Climate: A Guide to the Greenhouse Effect, 1989.)

the atmosphere by increasing the content of the carbon dioxide. For a long time, this hypothesis was accepted as obviously true and was applied to atmospheric phenomena practically without verification (Budyko, 1997; Greenhouse Effect, 1989; Green Peace Report, 1993).

\section{Alarming Warnings}

Increasing concentrations of greenhouse gases may accelerate climatic changes. Environmentalists predict that the global surface temperature can rise $1-4.5^{\circ} \mathrm{F}$ in the next 50 years and $2.2-10^{\circ} \mathrm{F}$ in the next century (Kyoto Protocol, 1997). Because of the rising temperature, evaporation will increase, which, in turn, will increase global precipitation. The intense rainstorms around the world will become more frequent. Ice melting will increase the global sea level, which will be likely to rise 2 feet in the next 50 years along the U.S. coast.

\section{Alarming Actions}

The above described changes in climate (if they occur) would profoundly affect the human environment, making people's lives uncomfortable and in some cases unbearable. That is 
why environmentalists called for large-scale actions aimed at a reduction of the emission of greenhouse gases (especially carbon dioxide) to the atmosphere. The rates and levels of the admissible emission for countries were defined in the frame of the Kyoto protocol of 1997, which required 5\% worldwide cuts in carbon dioxide emissions. The greatest damage would probably be done to the U.S. economy. To achieve the required carbon dioxide emission cuts by the year 2012, the U.S. would have to reduce its projected 2012 energy use by $25 \%$. "Most economic studies indicate that the cost of the Kyoto carbon dioxide emission cuts to the U.S. would amount to between $\$ 100$ billion and $\$ 400$ billion per year" (Baliunas, 2002).

\section{Theoretical Developments and Mathematical Models}

Huge potential damage to the world economy and western lifestyle led to revision and subsequent serious critique of the disastrous predictions made in the studies on global warming (Robinson et al., 1998; Sorokhtin, 2001; Baliunas, 2002). Thorough examination of the alarming predictions showed that until recently the theory of the greenhouse effect did not exist and all the numerical calculations and predictions were based on simplified mathematical models with rough estimates of the important parameters.

One of the simplified one-layer models was presented by Dr. Z. Mester (1996). In this one-layer model, atmosphere is considered to be confined by 2 imaginary surfaces: bottom (representing the Earth's surface absorbing and reradiating the solar radiation back to space) and top (representing the reflecting properties of Earth's atmospheric system or Earth's albedo ${ }^{1}$ ). Transformation of the solar energy $F_{S}$ by Earth's atmospheric system is shown in Figure 4. The effective temperature of the planet $T_{e}$ and solar radiation $F_{s}$ at the distance of Earth from the sun are linked by the Stefan-Boltzmann Law (Landau and Lifshits, 1979):

$$
F_{s}=4 \sigma\left(T_{e}\right)^{4},
$$

where $F_{S}$ is solar radiation energy flux (called the solar constant; for Earth $F_{s}=1.367 \times$ $\left.10^{6} \mathrm{erg} / \mathrm{cm}^{2} \mathrm{~s}\right), \sigma$ is the Stefan-Boltzmann constant, and $T_{e}$ is the absolute radiation temperature measured in ${ }^{\circ} \mathrm{K}$.

In Figure 4, the part $A(0<A<1)$ of this radiation determined by the planet's albedo is reflected by Earth's atmospheric system back to space; the part $B(0<B<1)$ of the radiation $F_{s}(1-A)$ penetrating the atmosphere is being absorbed by the atmosphere; and the part $F_{S}(1-A)(1-B)$ reaches the Earth's surface. This radiation is converted to energy in the infrared range behaving as black body radiation. The infrared radiation from the Earth's surface is partially absorbed $(f, 0<f<1)$ by greenhouse gases in the atmosphere, and the molecules of these gases reradiate this energy uniformly in all directions, thus warming the atmosphere.

Under these suppositions, the energy flux balance for the top of the atmosphere (Figure 4) is expressed by the following equation (Mester, 1996):

$$
F_{S}(1-A)(1-B)=(1-f) 4 \sigma\left(T_{0}\right)^{4}+4 \sigma\left(T_{A}\right)^{4} .
$$

The energy flux balance for the bottom of the atmosphere is expressed by the following equation:

$$
F_{S}(1-A)(1-B)+4 \sigma\left(T_{A}\right)^{4}=4 \sigma\left(T_{0}\right)^{4}
$$

\footnotetext{
${ }^{1}$ The fraction of solar radiation that is reflected back into space.
} 


\section{Greenhouse Effect “One-Layer Model”}

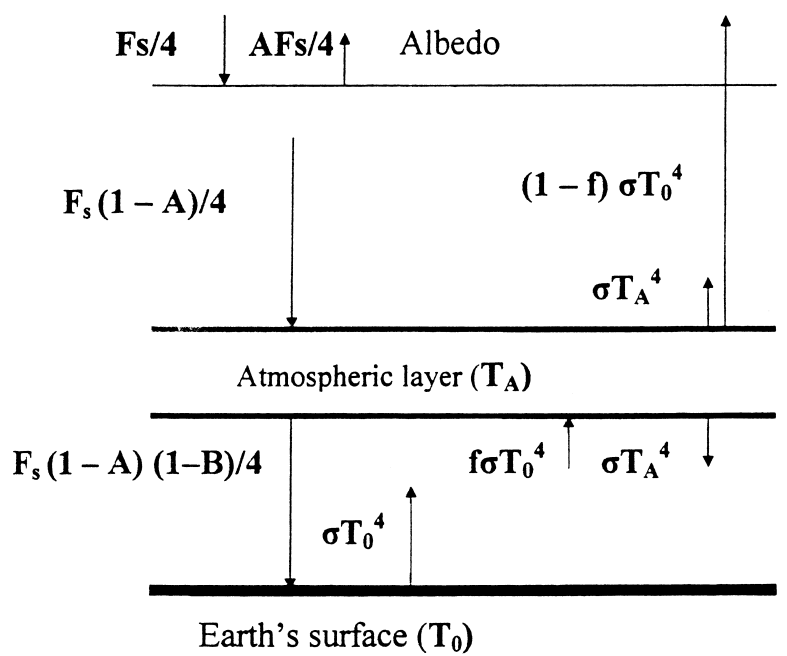

Figure 4. One-layer radiation model of the greenhouse effect. (Modified from Mester, 1996.)

Excluding $T_{A}$ from the last 2 equations and solving the resulting equation for $T_{0}$, one obtains a formula for the global Earth's surface temperature:

$$
T_{0}=\left[F_{S}(1-A)(2-B) / 8 \sigma(1-f / 2)\right]^{1 / 4} .
$$

It is difficult to give a physical interpretation of the variables of this model. What is the surface temperature, for example? In this model, it refers to the entire "atmospheric layer." It is not simple to evaluate parameters $A, B$, and $f$ for specific environmental conditions, but it is easy to play with this model considering typical climatic scenarios. Equation (4) allows for fast calculation of the "Earth surface temperature" for various values of the parameters $A, B$, and $f$, which represent Earth's albedo, atmospheric attenuation of the solar radiation, and the greenhouse effect, respectively. Several possible scenarios are presented in Table 1.

Table 1

Global warming scenario analysis (after Mester, 1996)

\begin{tabular}{lccccc}
\hline Scenario & $F_{S}, \mathrm{Wm}^{-2}$ & $A$ & $B$ & $f$ & $T_{0},{ }^{\circ} \mathrm{K}$ \\
\hline Existing & 1370 & 0.28 & 0.10 & 0.77 & 286 \\
3\% more greenhouse gas absorption & 1370 & 0.28 & 0.10 & 0.79 & 287 \\
1\% increase in solar activity & 1384 & 0.28 & 0.10 & 0.77 & 287 \\
No greenhouse gases & 1370 & 0.28 & 0.10 & 0.0 & 254 \\
Maximum greenhouse effect & 1370 & 0.28 & 0.10 & 1.0 & 302 \\
\hline
\end{tabular}


The results in Table 1 are obtained from the model counting on the heat transfer in atmosphere by radiation only. It is worth noting that the heat transfer by radiation dominates in the upper diffuse layers of stratosphere, mesosphere, and thermosphere only. In the meantime, Earth has a relatively dense atmosphere and the heat transfer in its lower, denser, layer (troposphere) mostly occurs by convection (Sorokhtin, 2001). Indeed, in the troposphere, with the air pressure exceeding $0.2 \mathrm{~atm}$, heat transfer by convection is dominant. When the temperature of a given mass of air increases, its volume increases by the same factor. As the hot air expands, it becomes less dense and rises. In turn, the denser cooler air drops and replaces the warmer air (Figure 5). This system works in the Earth's atmospheric system as a continuous surface cooler. The cooling effect of the air convection in the troposphere can considerably surpass the warming of the greenhouse effect.

The most important conclusion from this observation is that the temperature distribution in the troposphere has to be close to adiabatic, taking into consideration the fact that the air mass expands and cools while rising and compresses and heats while dropping (Sorokhtin, 2001). This last hypothesis was indirectly verified by a comparison of theoretical and experimental distributions of the temperature in the troposphere of the Earth (and Venus) that was done by Sorokhtin and Ushakov (1999).

For adiabatic transformation, the temperature dependence on gas pressure can be presented in the following form (Landau and Lifshits, 1979):

$$
T=C^{\alpha} p^{\alpha},
$$

where $C^{\alpha}$ is some constant that is defined separately.

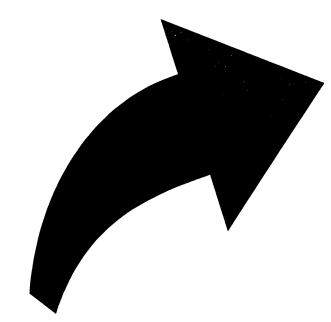

Warmer air rises

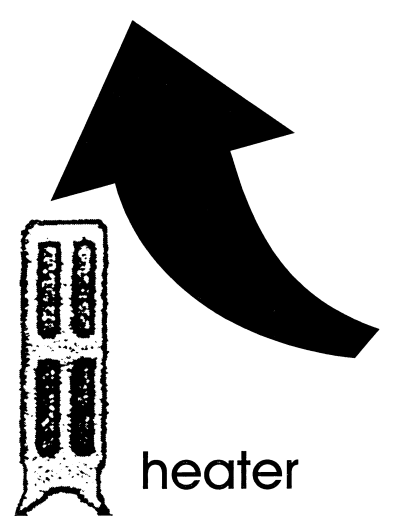

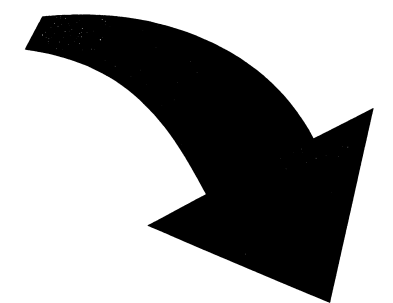

Cooler air drops and replaces the warmer air

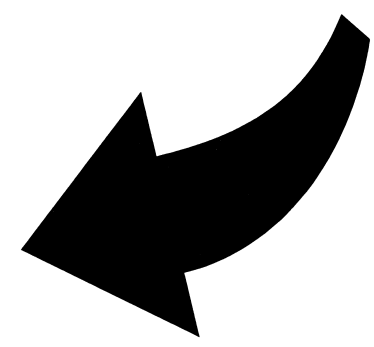

Figure 5. Schematic diagram of the heat transfer in the atmosphere by convection. 
The exponent $\alpha$ in Equation (5) is defined by

$$
\alpha=\frac{\left(C_{p}-C_{V}\right)}{C_{p}}=\frac{1-C_{p}}{C_{V}},
$$

where $C_{p}$ and $C_{V}$ are the thermal capacities of the gas under constant pressure and for a constant volume, respectively. For $\mathrm{CO}_{2}$ and $\mathrm{H}_{2} \mathrm{O}, \alpha=0.2308$; whereas for $\mathrm{O}_{2}$ and $\mathrm{H}_{2}$, $\alpha=0.2857$. Water vapor condensing in the troposphere emits the heat that increases the air temperature. Because of that, the adiabatic exponent $\alpha$ decreases. For example, for the humid Earth's troposphere $\alpha=0.1905$ (Sorokhtin and Ushakov, 1999).

Water vapor condensing in troposphere begets cloudiness, which is a leading factor that determines the Earth's albedo. This forms strong negative feedback between the solar radiation and the Earth's surface heating, which stabilizes the troposphere temperature. Under these circumstances, one can assume that the troposphere temperature is a linear function of the effective radiation temperature (temperature of an absolutely black body at a distance from the sun to the Earth). Combining this condition with Equation (5), one can write a formula that determines the "average Earth's surface temperature" at any altitude of the troposphere as an exponential function of the pressure at the same altitude:

$$
T(h)=b T_{e} p^{\alpha}(h),
$$

where $p(h)$ is the pressure of the atmosphere at altitude $h, T(h)$ is the "average surface temperature" at altitude $h, T_{e}$ is the effective radiation temperature, and $b$ is a scaling coefficient.

For Earth, $T_{e}=278.8^{\circ} \mathrm{K}$; contemporary temperature at sea level is $288^{\circ} \mathrm{K} ; T(0)=$ $288^{\circ} \mathrm{K}$; and the atmospheric pressure $p(0)=1 \mathrm{~atm}$. Substituting these data into Equation (7) $(288=b \times 278.8 \times 1)$, one can determine the scaling coefficient $b(b=1.033)$ and rewrite Equation (7) into the following more convenient form:

$$
T(h)=1.033 T_{e} p^{\alpha}(h),
$$

or

$$
T(h)=288 p^{\alpha}(h),
$$

which allows one to construct the temperature distribution for the troposphere layer of the atmosphere (if the exponent $\alpha$ is known). With such an approach, the focus of modeling is shifted to the definition of parameter $\alpha$, which determines the adiabatic heat transfer in the troposphere.

Sorokhtin and associates computed the values of $\alpha$ for typical conditions of the troposphere: for the absolutely dry and transparent troposphere $\alpha=0.286$; for the humid, heat-absorbing troposphere $\alpha=0.1905$ (Sorokhtin and Ushakov, 1999). The temperature distributions in the troposphere for these 2 conditions are shown in Figure 6. In this figure, one can see that for the same atmospheric pressure the near surface temperature of the dry and transparent troposphere is always greater than the near surface temperature for the humid, heat-absorbing troposphere.

Heat absorption by the greenhouse gases causes the further increase in this difference. This leads to the paradoxical (at first sight) conclusion that absorption of infrared radiation of the Earth does not increase, but, on the contrary, decreases the near surface temperature of the Earth. Using the adiabatic model, the explanation becomes simple: 


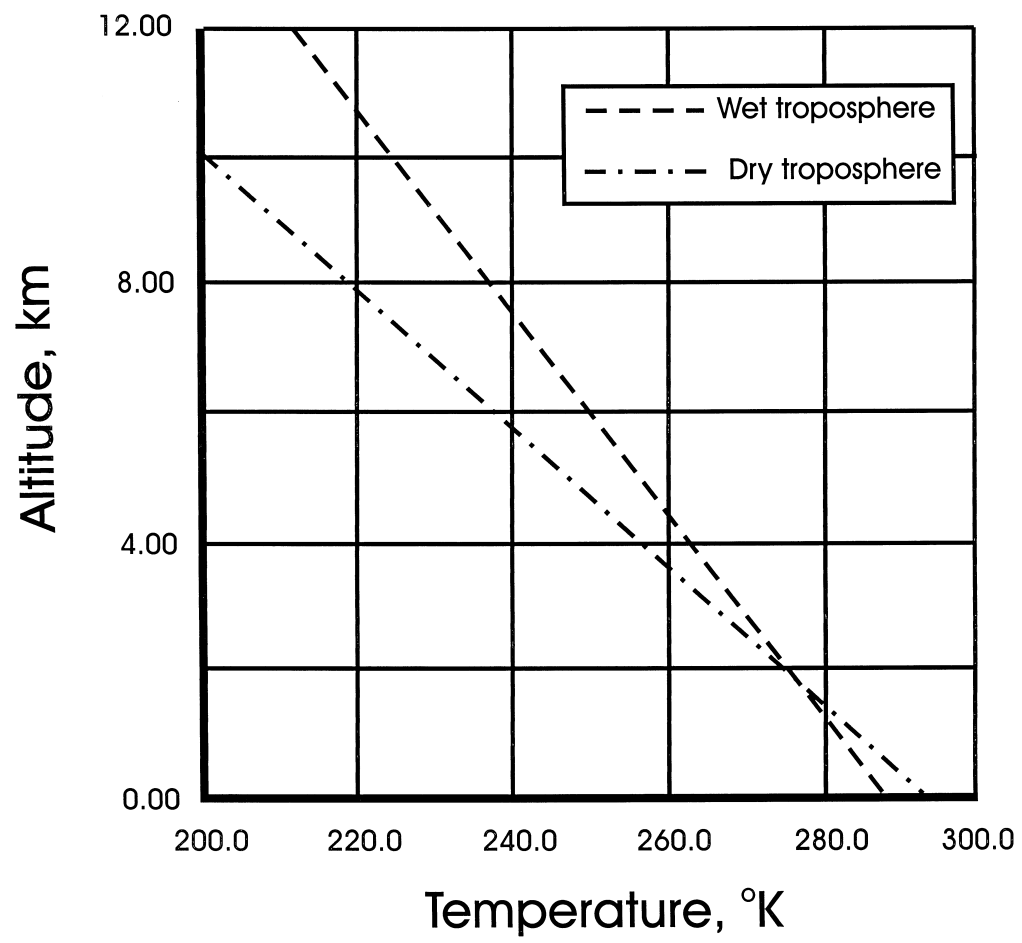

Figure 6. Adiabatic temperature distribution in the troposphere for the dry troposphere $(\alpha=0.286)$ and wet troposphere $(\alpha=0.1905)$. (Modified from Sorokhtin, 2001.)

with an increasing effective thermal capacity of the air (which occurs if the emission of the greenhouse gases to the atmosphere increases), the adiabatic exponent $\alpha$ decreases and the atmospheric temperature decreases (in accordance with Equation (8)).

A physical explanation of the phenomenon refers to high efficiency of the heat transfer by convection from the planet surface to the lower layers of the stratosphere from which heat is rapidly emitted to space by radiation. The adiabatic model was tested by Sorokhtin (2001) by comparing theoretical and experimental temperature distributions for the tropospheres of Earth and Venus, which demonstrated good agreement between the theory and experimental data $(0.1 \%$ accuracy for Earth and $1 \%$ accuracy for Venus). The adiabatic model shows that the Arrhenius hypothesis on global atmospheric warming as a result of the increased emission of greenhouse gases to the atmosphere is not true and must be revised.

The major flaw of the radiation theory of global warming is that it completely ignores the heat transfer in the atmosphere by convection. Of the total heat transfer from the Earth's surface to the troposphere, convection accounts for 67\%, radiation accounts for $11 \%$, and the condensation of water vapor in the troposphere accounts for $22 \%$ (Sorokhtin, 2001).

\section{Effect of Man-Induced Carbon Dioxide Emission on the Earth's Climate}

The concentration of $\mathrm{CO}_{2}$ in the Earth's atmosphere has been increasing in the 20th Century, as shown in Figure 2. The carbon content in the atmosphere was increasing 
by approximately 3 billion tons per year at the end of the century. The rate of the total man-induced $\mathrm{CO}_{2}$ emission to the Earth's atmosphere is currently about 5-7 billion tons per year (Schimel, 1995; Robinson et al., 1998). This huge amount of carbon dioxide not only changes the gas composition of the atmosphere but also slightly increases the atmospheric pressure.

One can use the adiabatic model together with the sensitivity analysis (Khilyuk et al., 1994; Katz et al., 1996) to evaluate the effect of human emissions of carbon dioxide on the global temperature. Taking logarithms from both sides of Equation (9) results in

$$
\ln T=\ln 288+\alpha \ln p .
$$

Introducing notation $A(\alpha, p)$ for the right part of Equation (10), one can find the sensitivity functions of $\ln T$ as partial derivatives of $A(\alpha, p)$ with respect to parameters $\alpha$ and p. Differentiating Equation (10), one obtains

$$
\frac{1}{T} d T=\frac{\partial A(\alpha, p)}{\partial \alpha} d \alpha+\frac{\partial A(\alpha, p)}{\partial p} d p .
$$

Substituting partial derivatives of $A(\alpha, p)$ and multiplying both parts of Equation (11) by $T$, the following equation is obtained:

$$
d T=T\left(\ln p d \alpha+\frac{\alpha}{p} d \alpha\right)
$$

Transition to finite differences in differential Equation (12) yields

$$
\Delta T \approx T \ln p \Delta \alpha+T \frac{\alpha}{p} \Delta p
$$

Equation (13) is convenient for the analysis of the effect of man-induced emissions of greenhouse gases on the global temperature. If, for example, the concentration of $\mathrm{CO}_{2}$ in the atmosphere increases 2 times (from $0.035 \%$ to $0.07 \%$ ), which is expected by the year 2100 , then the pressure will increase by $\Delta p=1.48 \times 10^{-4}$ atm and $\Delta \alpha=-4 \times 10^{-6}$ (Sorokhtin, 2001). At sea level, if the pressure is measured in atmospheres, then $p=1$ and $\ln p=0$. Thus,

$$
\Delta T \approx T \alpha \Delta p
$$

After substitution of $T=288^{\circ} \mathrm{K}, \alpha=0.1905$, and $\Delta p=1.48 \times 10^{-4}$ atm into Equation (14), one finds $\Delta T \approx 8.12 \times 10^{-3 \circ} \mathrm{K}$. Thus the increase in the surface temperature at sea level caused by the man-induced $\mathrm{CO}_{2}$ emissions will be $<0.01^{\circ} \mathrm{K}$, which is negligible compared to the natural temporal fluctuations of the global temperature.

This increase will be slightly higher at higher altitudes, where the pressure is $<1$ atm and its logarithm is negative. Multiplication of $\ln p$ by the negative value of $\Delta \alpha$ will increase $\Delta T$ (the global temperature change; Equation (13)). If, for example, $h=10 \mathrm{~km}$, then the barometric pressure $p \approx 0.24$ atm and the temperature is about $200-220^{\circ} \mathrm{K}$ (FMH-1, 1995). Substituting all necessary data $\left(T=220^{\circ} \mathrm{K}, p \approx 0.24\right.$ atm, $\ln p \approx$ $-1.4286, \Delta p=1.48 \times 10^{-4}$ atm, $\alpha=0.1905, \Delta \alpha=-4 \times 10^{-6}$ into Equation (13), one obtains $\Delta T \approx 2.710 \times 10^{-2 \circ} \mathrm{K}$, which is $<0.03^{\circ} \mathrm{K}$.

From these estimates one can deduce a very important practical conclusion that even a considerable increase in the man-induced emission of carbon dioxide and other greenhouse gases practically does not change the global atmospheric temperature. Thus the 
hypothesis of current global warming as a result of increased greenhouse gas emissions into the atmosphere is a myth. Humans are not responsible for the increase in the global surface temperature of $1^{\circ} \mathrm{F} \approx 0.56^{\circ} \mathrm{K}$ during the last century, and the main culprit is our sun, which changes the Earth's climate because of varying radiation.

\section{Do Greenhouse Gases Constitute Cause or Effect of Global Warming?}

Defenders of the greenhouse global warming theory usually point out that increased emissions of the greenhouse gases is accompanied by the increase in global temperature, and, consequently, the increased emissions cause the global warming. Yet an increase in carbon dioxide concentration closely follows a 300 year warming trend. "The observed increases are of a magnitude that can, for example, be explained by oceans giving off gases naturally as temperatures rise. Indeed, recent carbon dioxide increases have shown a tendency to follow rather than lead global temperature increases" (Robinson et al., 2001).

Figure 7 shows that the global surface temperature is directly correlated with solar activity. The greater the solar activity, the greater the sun's radiation, and, consequently,

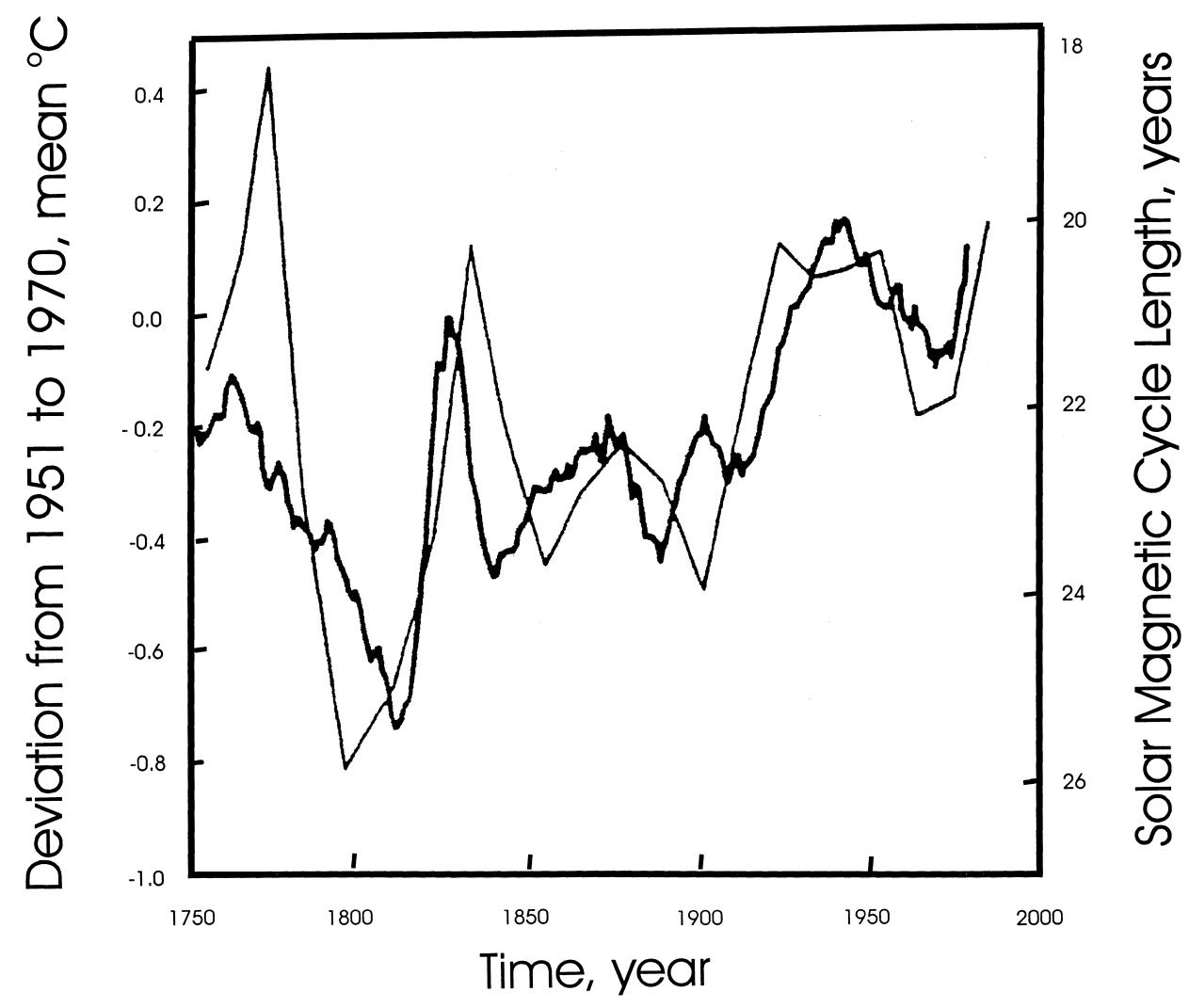

Figure 7. Moving 11 year average terrestrial temperatures (Northern Hemisphere) shown as deviations in ${ }^{\circ} \mathrm{C}$ from the $1951-1970$ mean value (left vertical axis and thick line) and the solar magnetic cycle lengths (right vertical axis and thin line). (Modified from Robinson et al. (1998, Figure 3), Jones (1986), Grovesman and Landsberg (1979), and Baliunas and Soon (1995).) 
the Earth's surface absorbs more radiation from the sun. This leads to an increase in the surface temperature and a consequent warming of the atmosphere by convection, water vapor condensation, and radiation. Figure 8 shows that on the large historical scale the surface temperature deviations lead to the corresponding changes in the atmospheric carbon dioxide concentrations. Apparently the temperature changes drive the changes in the carbon dioxide concentration: the higher the temperature, the greater the concentration of carbon dioxide (Sorokhtin, 2002; Robinson et al., 1998).

When the temperature increases, the solubility of the carbon dioxide in the ocean water decreases, and, as per the Henry Law, part of the carbon dioxide of the ocean water is transferred into the atmosphere to restore a disturbed dynamic equilibrium between the carbon dioxide contents of the ocean water and the atmosphere. Contemporary oceanic water contains 50-60 times more carbon dioxide than atmospheric air. Because of the inverse dependence of the $\mathrm{CO}_{2}$ solubility in the ocean water on temperature, the increase in temperature will cause an increase in the atmospheric $\mathrm{CO}_{2}$ concentration.

Similarly, during the Devonian and Silurian times, for example, the atmospheric $\mathrm{CO}_{2}$ pressure was high because of prevailing high temperatures. Lush vegetation prevailed,
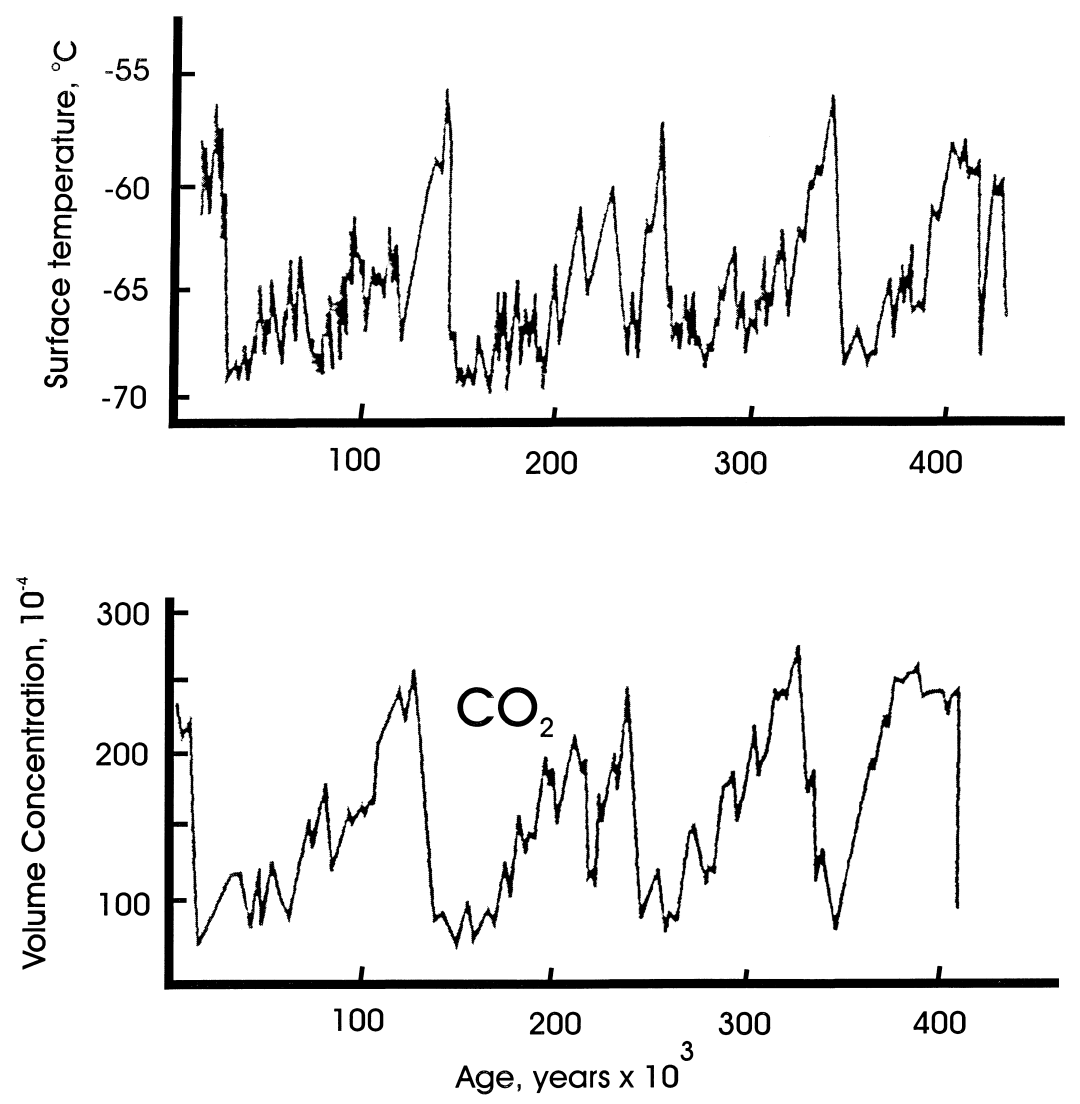

Figure 8. Correlation of the changes in concentrations of carbon dioxide with the changes in air temperature (upper graph) for the last 420 thousand years at the Antarctic station "Vostok." Data were obtained from the well logs by drilling in ice up to a depth of 3,623 $\mathrm{m}$. (Modified from Sorokhtin (2001, Figure 3) and Kotlyakov and Danilov (1999).) 


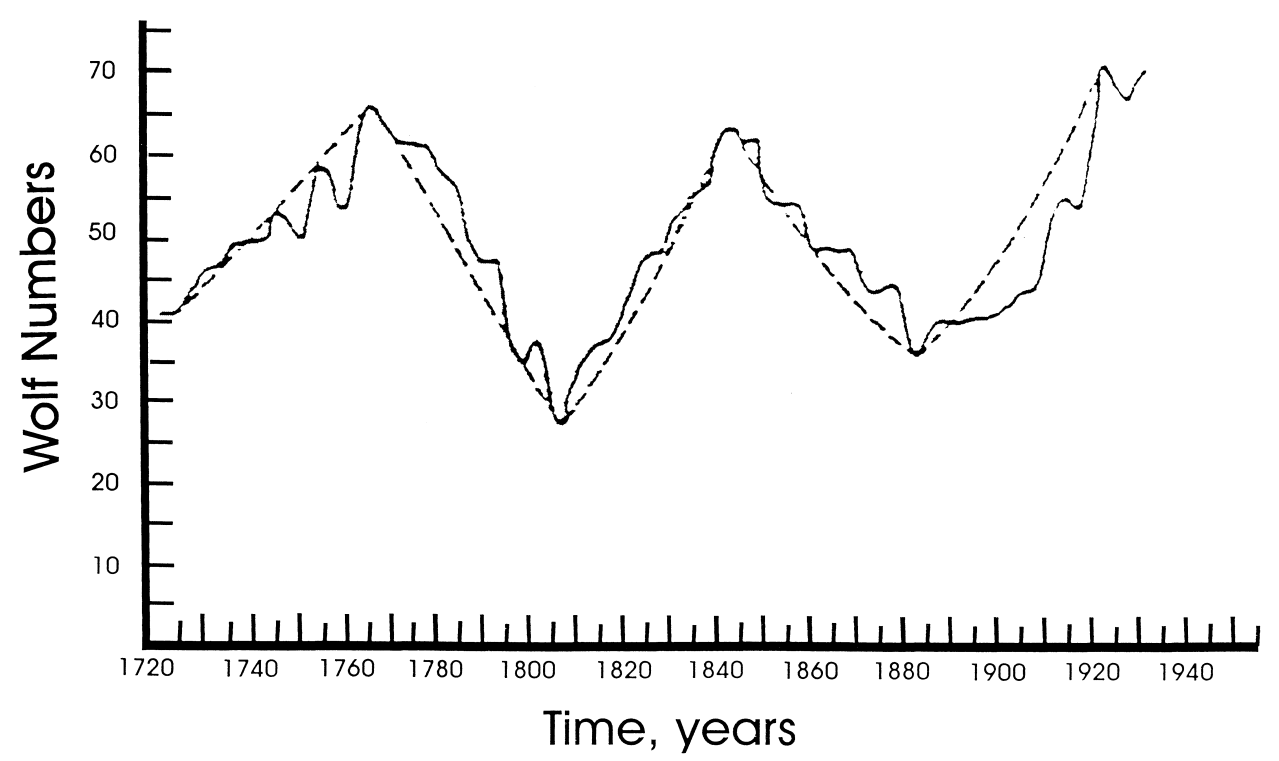

Figure 9. Ninety year cycles of solar activity. (Modified from Sarafyan (2000, p. 18, Figure 3).)

and the $\mathrm{Ca} / \mathrm{Mg}$ ratio of sea water was low, with abundant dolomite formation (Chilingar, 1956). Thus the $\mathrm{Ca} / \mathrm{Mg}$ ratio can provide valuable indirect information on the atmospheric concentration of $\mathrm{CO}_{2}$.

An analogous situation could be observed in the warm Cretaceous period when the temperature of the world ocean was about $15^{\circ} \mathrm{C}$ higher than the present one. Estimates by Sorokhtin and Ushakov (1991) showed that the $\mathrm{CO}_{2}$ partial pressure in the atmosphere was about 1.7-2 times higher than the present one. This increase in the atmospheric $\mathrm{CO}_{2}$ content, however, was caused by the temperature increase and not vice versa. Obviously, this increase could not be attributed to human activity.

A rising trend in the atmospheric $\mathrm{CO}_{2}$ concentration (Figure 2) is the effect of increasing global temperature that most probably can be attributed to increased solar radiation in the rising phase of the latest 80-90 year cycle of solar activity (see Figure 9). Thus another cooling down period will follow. Existence of these cycles has been acknowledged by all the scientists who have studied this phenomenon over the last hundred years (Sarafyan, 2000).

\section{Discussion}

Based on the analysis presented above, the authors conclude that the man-induced emission of carbon dioxide and other greenhouse gases has a very small effect on global atmospheric warming. Experimental data and the adiabatic model show that it is the rising global temperature that causes an increase in the atmospheric concentration of carbon dioxide and not vice versa. Because of this, the authors abandon the popular environmental doctrine of global warming that an increase in man-induced emissions of greenhouse gases heats the atmosphere.

Unfortunately, speculations on global warming were used as a "justification" for important political decisions. For example, in July of 2002 in California, Governor G. 
Davis signed the global warming control bill, which requires a dramatic cut in vehicle emissions of greenhouse gases. This would mean higher production costs for automakers and higher prices for consumers.

Thus the following question arises: should developed countries reduce their carbon dioxide emissions in compliance with the Kyoto Protocol? The answer is no. First, because the effects of these cuts will be negligible. Second, because these cuts will result in economic disaster for developed countries (first of all for the U.S.), causing huge economic losses and job cuts. On July 11, 2002, James L. Connaughton, the U.S. President's Advisor and the Chairman of the White House Council of Environmental Quality, told senators that withdrawal from this international treaty would preserve billions of dollars and millions of jobs. He stated that "the Kyoto Protocol would have cost our economy up to $\$ 400$ billion (a year) and caused the loss of up to 4.9 million jobs, risking the welfare of the American people and American workers." Instead, the President's advisors promised to develop and publish in March of 2003 a comprehensive research plan on the problem of global warming (Los Angeles Times, July 12, 2002).

Discussing consequences of possible emission cuts, one should take into consideration that the increased emissions of carbon dioxide and water vapor are positive factors for agriculture and biological protection. "In light of some of the hysterical language surrounding the issue of greenhouse gases, it is also worth noting that carbon dioxide, the primary greenhouse gas produced by burning fossil fuels, is not a toxic pollutant." On the contrary, it is essential to life on Earth. "Agricultural experts estimate a ten percent increase in crop growth in recent decades-due directly to the fertilization effect of increased carbon dioxide in the air" (Baliunas, 2002).

The cyclic processes in nature are common phenomena. In addition to solar cycles, one can mention atmospheric, oceanic, and sedimentation cycles. In the evolutionary system, cyclic changes manifest a self-organizing process of search for the system equilibrium as a system reaction to external disturbance (Sarafyan, 2000). People must adapt themselves to these cycles and learn how to live with them.

In this context, the Caspian Sea level "story" is very instructive (Sorokhtin, 2001). After many years of steep decline (1980-1990), the sea level began rising at a threatening rate. In the meantime, intense speculations about the sea drying out as a result of human activities resulted in large-scale research and development on the project of redirection of the Northern Russian rivers into the Volga River. If the Soviet Union did not collapse in 1991, this project would have been implemented, creating enormous ecological problems to the dense population of the Volga River Basin. This example must teach us not to rush when dealing with a large-scale natural phenomenon.

\section{Conclusions}

The rising global temperature causes an increase in the concentration of carbon dioxide in the atmosphere. Carbon dioxide in the atmosphere absorbs part of the solar energy reradiated from the Earth's surface, resulting in the trapping of energy and the heating of the atmosphere. This heating is compensated for by the cooling effect of air convection, which transfers heat from the Earth's surface to the upper layers of the troposphere, from where it is radiated into space.

The adiabatic model adequately describes the heat transfer in the Earth's atmospheric system and together with sensitivity theory may be used for quantitative analysis of the temperature changes in the atmosphere. The effect of the increasing concentration of carbon dioxide caused by man-induced emissions is very small. Thus it can be neglected 
and should not be considered in making political decisions for the present or in the near future. It is not mankind but our sun that is responsible for the heating the Earth's atmosphere during the last $40-50$ years.

As a result of the increased temperature, $\mathrm{CO}_{2}$ from ocean water is transferred to the atmosphere. Thus one can see that "cause" and "effect" are reversed, as presented by proponents of the theory of global warming as a result of an increase in $\mathrm{CO}_{2}$ emissions. The writers congratulate the U.S. government for not signing the Kyoto Protocol.

\section{References}

Arrhenius, S. 1896. On the influence of carbonic acid in the air upon the temperature of the ground. Phil. Mag. 41:237-276.

Baliunas, S. L. 2002. The Kyoto protocol and global warming. Imprimis 31(3):1-7; http://www. hillsdale.edu.

Budyko, M. I. 1997. The Carbon Dioxide Problem. Sankt Petersburg: Gidrometeoizdat (in Russian).

Chilingar, G. V. 1956. Relationship between Ca/Mg ratio and geologic age. Bull. Am. Assos. Petrol. Geol. 40:2256-2266.

Chilingarian, G. V., and K. H. Wolf, eds. 1988. Diagenesis, I. Amsterdam: Elsevier.

EPA Global Warming Site: Climate; http://www.epa.gov/globalwarming/climate/index.html.

FMH-1. 1995. Federal Meteorological Handbook No.1; http://www.nws.noaa.gov/oso/oso1/oso12/ fmh1.

Greenhouse Effect, Changing Climate and Ecosystem. Leningrad: Gidrometeoizdat (in Russian).

Green Peace report. 1993. Global Warming, Moscow University, Moscow (in Russian).

Katz, S. A., L. F. Khilyuk, and G. V. Chilingar. 1996. Sensitivity analysis and multivariant modeling for formation pressure and temperature fields in inhomogeneous media. J. Petrol. Sci. Engr. 16:95-108.

Keeling, C. D., and T. P. Whorf. 1997. Trends Online: A Compendium of Data on Global Change, Carbon Dioxide Information Analysis Center, Oak Ridge National Laboratory; http://cdiac. esd.ornl.gov/ftp/ndp001r7/.

Khilyuk, L. F., S. A. Katz, G. V. Chilingarian, and F. Aminzadeh. 1994. Numerical criterion and sensitivity analysis for time dependent formation pressure in a sealed layer. J. Petrol. Sci. and Engr. 12:137-145.

Kondratiev, K. Ya. 1992. Global Climate. Moscow: Nauka (in Russian).

Kotlyakov, V. M., and A. I. Danilov. 1999. Global changes in Antarctic Continent. Earth and Universe 4:3-12 (in Russian).

Kyoto Protocol to the United Nations Framework Convention on Climate Change. 1997. http:// www.epa.gov/globalwarming/publications/reference/index. html.

Landau, L. D., and E. M. Lifshits. 1979. Statistical Physics. Moscow: Nauka (in Russian).

Marov, M. Ya. 1986. Planets of the Solar System. Moscow: Nauka (in Russian).

Mester, Z. S. 1996. Global Warming, A Brief Survey. Presented at the USC Energy and Environment Seminar, April 9.

Robinson, A. B., S. L. Baliunas, W. Soon, and Z. W. Robinson. 1998. Environmental effects of increased atmospheric carbon dioxide.

Sarafyan, N. 2000. Cycles and Self-Organization. Los Angeles, CA: Nubar Publishers.

Schimel, D. S. 1995. Global Change Biology 1:77-91.

Sorokhtin, O. G. 2001. Greenhouse effect: Myth and reality. Vestnik Russian Academy of Natural Sciences 1(1):8-21 (in Russian).

Sorokhtin, O. G., and Ushakov, S. A. 1999. Greenhouse effect and global evolution of the Earth climates. Izvestiya Russian Academy of Natural Sciences 3:82-101 (in Russian).

Waddel, K. L., D. D. Oswald, and D. S. Powell. 1989. Forest Statistics of the United States, 1987. U.S. Forest Service and Dept. of Agriculture; http://www.fs.fed.us/pl/rpa/89pub.htm. 
Copyright $\odot 2003$ EBSCO Publishing 